\title{
On the Diurnal Variation of Oxygen Content in Surface Layers of the Hydrosphere
}

\author{
by \\ Y. Sugiura \\ Meteorological Research Institute
}

(Received July 4, 1953)

\begin{abstract}
In eutropic lakes or coastal waters, a marked diurnal varia, tion of oxygen content is found. There are two types of variations. In one of them, a minimum occurs mostly at about $6 \mathrm{~h}$ and a maximum at about $17 \mathrm{~h}$. This type of variation is often seen in stable and homogeneous water masses. In the other type, minimum and maximum occur at different hours from the above-mentioned. In this case, the influence of diffusion and advection or convection upon the diurnal variation of oxygen content must be considered. In this paper, the former case is chiefly treated. In this case, the amplitude of the diurnal variation depends upon the intensity of radiation, the duration of insolation, water temperature, number and size of phytoplanktons, etc. Indeed, the amplitude is larger on a clear day than on a cloudy day and it is also larger in summer than in winter. It was found by calculation that the diurnal variation should have a minimum and a maximum at a certain time distance respectively before and after the noon. By using the data of the diurnal variations of dissolved oxygen, the amount of the oxygen production or consumption during a whole day is computed. Assuming a certain size and number of phytoplanktons, computed values agreed well with the observation.
\end{abstract}

\section{Introduction}

The main source of dissolved oxygen in surface layers in oceans and lakes is the absorption of the air in water as well as the production by photosynthesis. On the other hand, it is consumed by respiration by iving matter and oxidation of organic and inorganic substances. Furthermore, convection, advection and diffusion give a great influence on the oxygen content. As will be stated below, it was found by E. KurAshige [1], Y. MiYake and the present author that in the diurnal variation of oxygen content in the surface layers, minimum and maximum 
generally appeared at about $6 \mathrm{~h}$ and $17 \mathrm{~h}$ respectively in homogeneous waters. On the other hand, when flow and mixing of water exist, the shape of the diurnal variation becomes much complicated. Up to the present day, this problem has been treated only qualitatively. The present author intends to treat it quantitatively and to clarify the mechanism concerning the diurnal variation of oxygen content.

\section{Results of observation}

i) The diurnal variation of dissolved oxygen in homogeneous waters.

The curve A in Fig. 1 shows a result of observation of the dissolved oxygen in sea water carried out by the author and K. KAMEDA on the coast of Hachijo Island $\left(33^{\circ} 05^{\prime} \mathrm{N}, 139^{\circ} 48^{\prime} \mathrm{E}\right)$. In the curve $\mathrm{B}$ in Fig. 2 the diurnal variation of dissolved oxygen in well water in the island is given (Dec., 1952). Fig. 2 shows a result by E. Kurashige for the moat around the Imperial Palace, Tokyo. These results show that a maximum occurs at about 14 to $19 \mathrm{~h}$, and a minimum at about 5 to $8 \mathrm{~h}$.

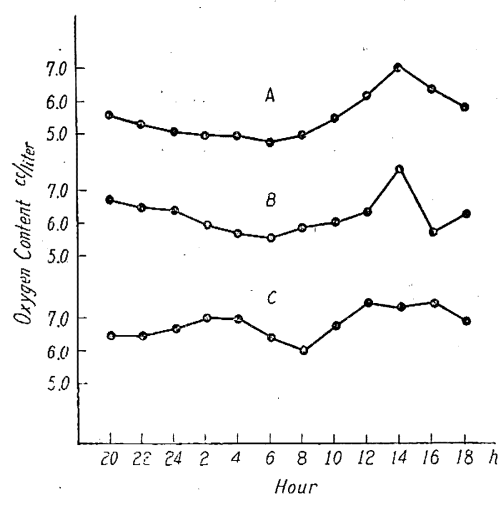

Fig. 1. The diurnal variation of dissolved oxygen, obtained on the coast of Hachijo Island (Dec., 1952).

ii) The diurnal variation of dissolved oxygen when flow or mixing exists.

The resuit shown in Fig. 3 was obtained in the Straits of Misaki at the end of Miura Peninsula. It has a maximum at $18 \mathrm{~h}$ and a minimum at. $3 \mathrm{~h}$. Dr.Y. Miyake [2] explained that this variation was brought about

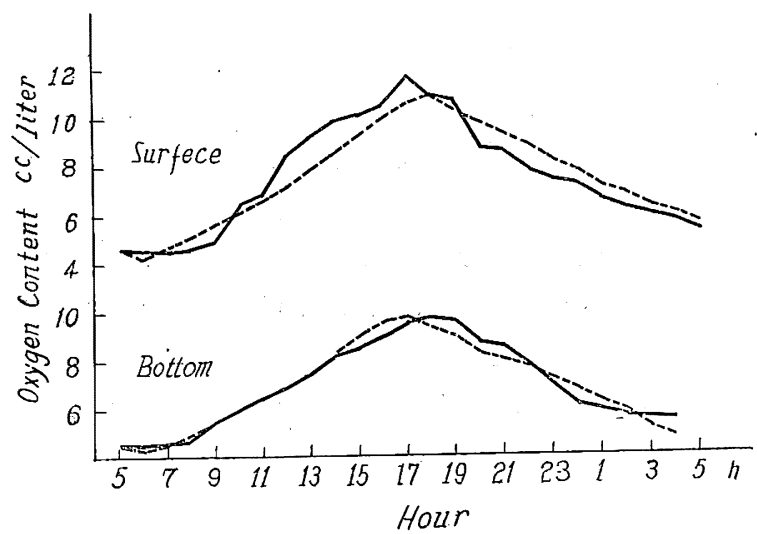

Fig. 2. The diurnal variation of oxygen content obtaired by E. Kurashige (April, 1932). Solid lines show che observed values and dotted lines the calculated values.

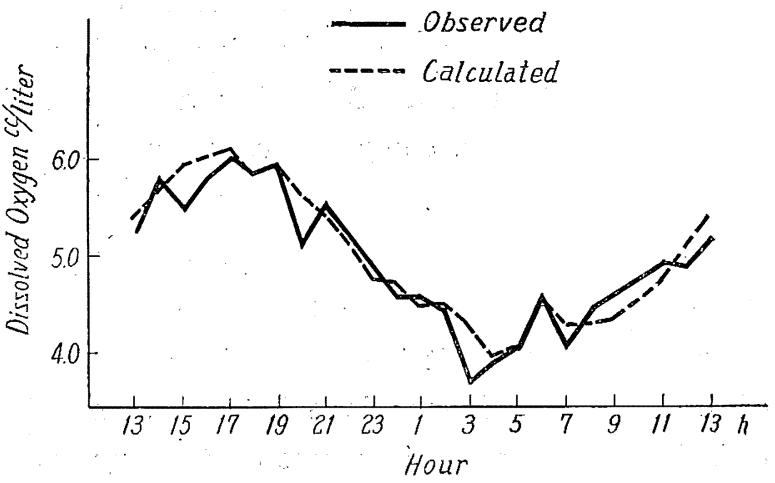

Fig. 3(a). The diurnal variation of dissolved oxygen obtained in the Straits of Misaki by Y. Miyake (Aug., 1947). 


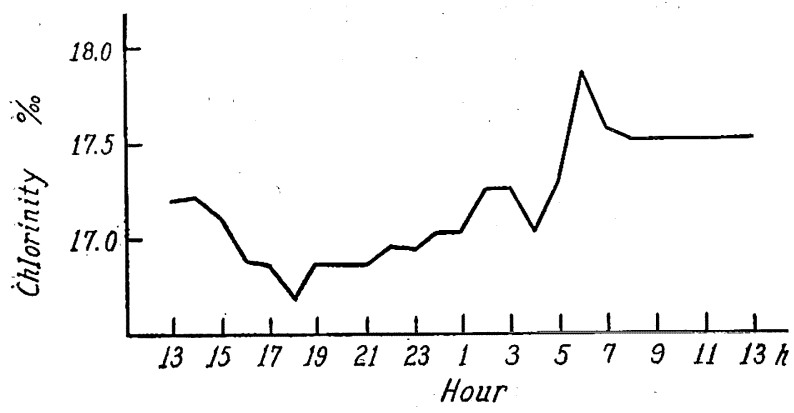

Fig. 3 (b). The diurnal variation of chlorinity in the straits of Misakif

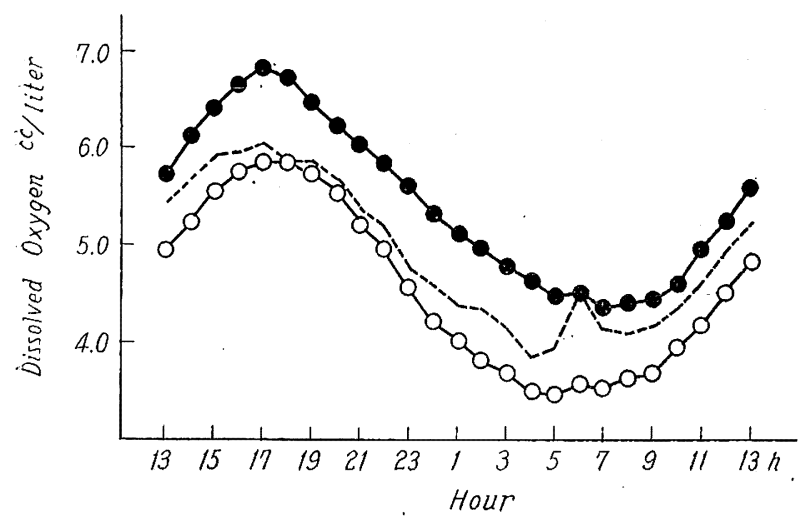

Fig. 3(c). The diurnal variation of dissolved oxygen in two water masses which mix with each other in the straits of Misaki. by the mixing of two kinds of water masses having different oxygen contents. $\mathrm{He}$ estimated each proper diurnal variation of oxygen content in two kinds of waters, and composed them determining the rate of mixing by means of the chlorinity.

The present author repeated the calculation concerning this problem. The author determined the shape of each proper diurnal variation of oxygen content in two kinds of waters, by use of the method mentioned below. Fig. 4 shows the isopleth of the diurnal variation of oxygen content obtained in a small pond (dia. $10 \mathrm{~m}$, depth $3 \mathrm{~m}$ ) in front of our laboratory in midsummer. It seems to show

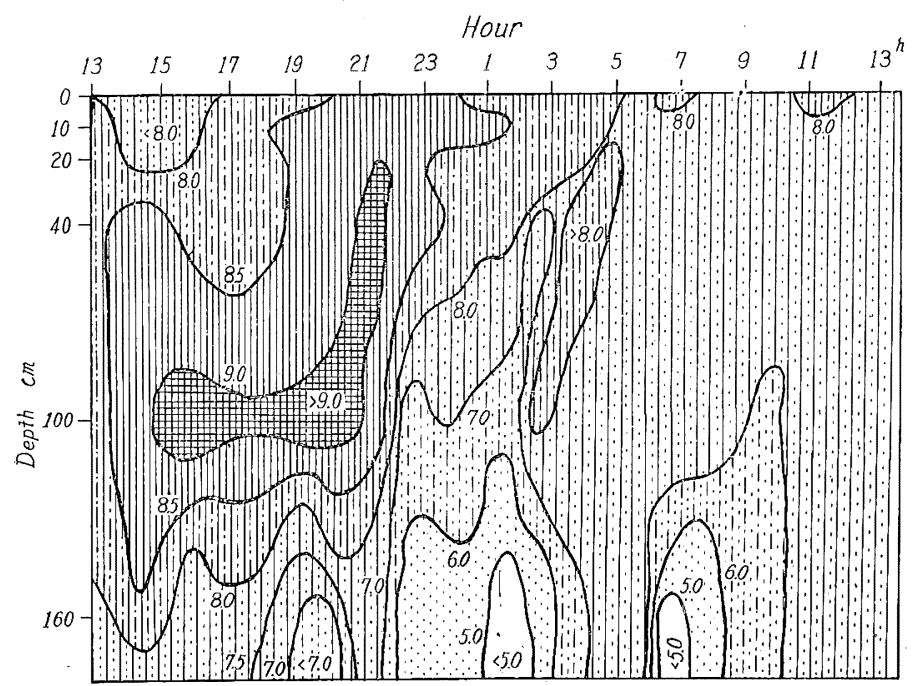

Fig. 4. The diurnal variation of oxygen observed in a pond of Meteorological Research Institute, Tokyo (Aug., 1952). 
the effect of conveciton and diffusion upon the diurnal variation. The curve in C Fig.1 shows the result of observation in a pool (dia. ca. $5 \mathrm{~m}$, depth $2 \mathrm{~m})$ supplied with spring water at the coast of Hachijo Island. There are two maxima in the curve $\mathrm{C}$, one of which appearing at midnight.

iii) The amplitude of the diurnal variation of dissolved oxygen.

Fig. 5[3] shows the diurnal variation in the degree of saturation obtained on the coast of Hachijo Island in summer and in winter respectively. In these results, it is found that the amplitude of diurnal variation is much larger on a clear day than on a cloudy day and also that it is larger in summer than in winter.

Results obtained by $\mathrm{E}$. Kurashige [4] given in Fig. 6 are quite similar to those in Fig. 5.

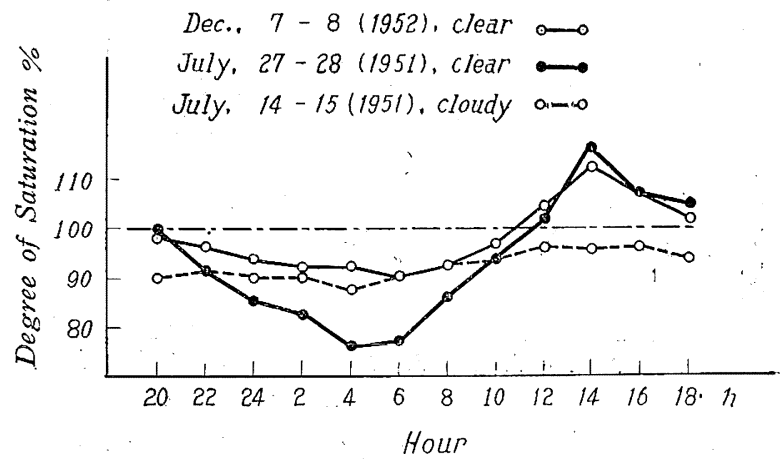

Fig. 5. The comparison "of amplitude of the diurnal variation.

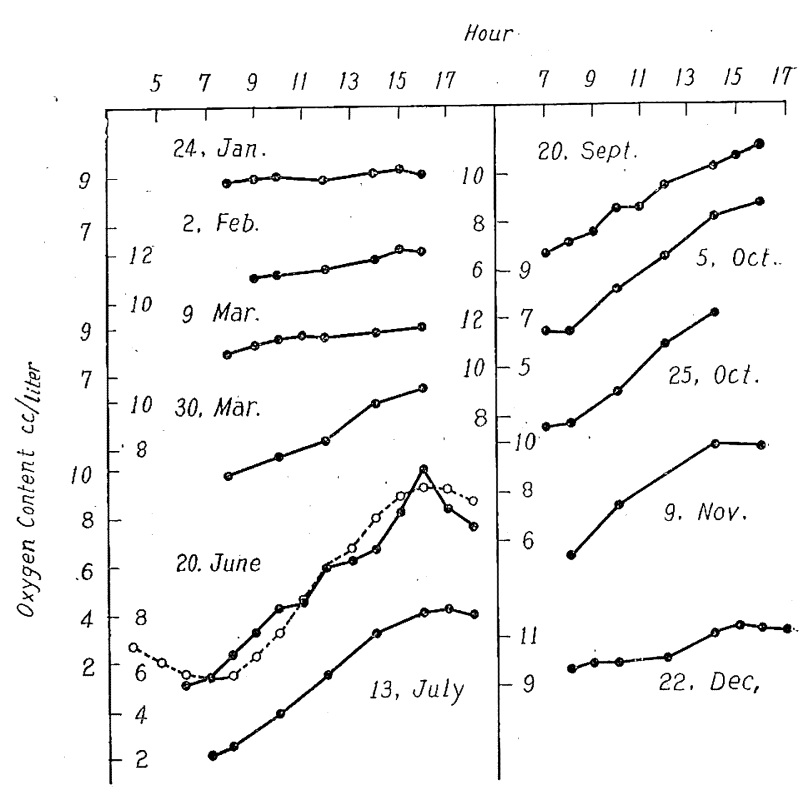

Fig. 6. The seasonal change of the diurnal variation of dissolved oxygen (after E. KurAshige) in the moat around the Imperial Palace, Tokyo.

\section{On the factors affecting the dissolved oxygen}

Main factors affecting the diurnal variation of dissolved oxygen are as follows:

1) photosynthesis

2) respiration

3) oxidation of organic and inorganic substances

4) diffusion

5) advection or convection.

Then, the time variation of oxygen content owing to these factors may be: expressed as follows : 
(1)

$$
\begin{aligned}
\frac{\partial S}{\partial t}= & B(x, y, z, t)+\frac{\partial}{\partial x}\left(A_{x} \frac{\partial S}{\partial x}\right)+\frac{\partial}{\partial y}\left(A_{y} \frac{\partial \dot{S}}{\partial y}\right)+\frac{\partial}{\partial h}\left(A_{h} \frac{\partial S}{\partial h}\right) \\
& -\left(v_{x} \frac{\partial S}{\partial x}+v_{y} \frac{\partial S}{\partial y}+v_{l} \frac{\partial S}{\partial \hbar}\right)-S\left(\frac{\partial v_{x}}{\partial x}+\frac{\partial v_{y}}{\partial y}+\frac{\partial v_{h}}{\partial h}\right),
\end{aligned}
$$

where

$$
\begin{aligned}
& B=P-R, \\
& R=R_{1} e^{k_{1}}+R_{2} e^{k_{2} \theta}, \\
& P=f I \pi r^{2} n \varepsilon Q a e^{k \theta}, \\
& I=I_{0} \gamma \cos Z e^{-\alpha \sec } Z^{-\beta a} .
\end{aligned}
$$

In the above formulae, the following notations are used.

$S$ : oxygen content

$I_{0}:$ the solar constant

$\alpha$ : the turbidity coefficient of light in water

$\beta:$ the extinction coefficient of light in water

$d$ : the depth from the surface to the observed layer

$\gamma$ : a fraction of the total light from the sun and sky entering into the inside of water on a clear day

$Z$ : the sun's zenith angle

$P$ : the amount of oxygen produced at $0^{\circ} \mathrm{C}$ by photosynthesis in a unit volume at a certain layer

$k$ : the temperature coefficient of photosynthesis

$R_{1}, R_{2}$ : the amount of oxygen consumed at $0^{\circ} \mathrm{C}$ respectively by respiration of living matter and by oxidation of organic and inorganic substances in a unit volume at a certain layer

$\theta: \quad$ water temperature

$k_{1}, k_{2}$ : the temperature coefficients respectively of respiration and of oxidation

$A$ : the coefficient of eddy diffusion

$v:$ the velocity of flow

$r$ : the mean radius of phytoplanktons

$n$ : number of phytoplanktons in a unit volume of water

$Q$ : the apparent quantum yield of oxygen

$a$ : rate of absorption of light by a phytoplankton

$f:$ the photochemical equivalent, $0.18 \mathrm{cc} \mathrm{O}_{2} / g$ cal which is obtained by the well-known energy bilanz, i.e.,

$6 \mathrm{CO}_{2}+6 \mathrm{H}_{2} \mathrm{O}=\mathrm{C}_{6} \mathrm{H}_{12} \mathrm{O}_{6}+6 \mathrm{O}_{2}-741.5 \mathrm{~K} \mathrm{cal}$

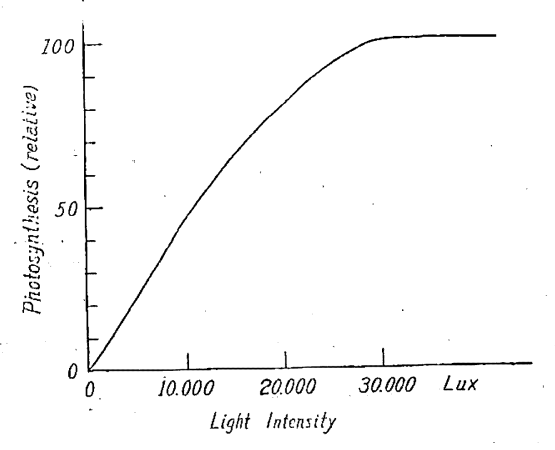

Fig. 7. The relation between the intensity and the relative photosynthesis (after NIELSEN, E. S., 1952). 
$\varepsilon:$ a parameter introduced in the following way.

Fig. 7 shows the relation between the relativa photosynthesis $Q a I$ and light intensity $I$ at constant temperature. In this figure, it is linear within a certain range of weaker light intensity. $\varepsilon$ denotes the ratio of the apparent quantum yield of oxygen correspoding to the strong light intensity, to that in the linear part.

Then,

$\pi r^{2} n$ : the illuminated total surface area of phytoplanktons suspended in a unit volume of water

$Q a e^{k \theta}:$ a fraction of incident light energy used for photosynthesis

For the convenience of calculation we may put,

$$
g=\gamma \cos Z e^{-\alpha \sec Z},
$$

where $g$ denotes the quotient of light energy below the water surface divided by the solar constant.

$$
I_{\varepsilon}=I \varepsilon e^{k \cdot \theta},
$$

and

$$
p=f Q a \pi r^{2} n
$$

where $p$ depends upon number, size and other physico-chemical properties of phytoplanktons.

The relation between $p$ and $P$ is,

$$
P=p I_{\varepsilon}
$$

\section{On the diurnal variation of oxygen content in the moat around the Imperial Palace}

Observation was made on the 4th and 5 th of April, 1932, by E. Kurashige, respectively in surface and bottom layers. The depth of the moat is about $1 \mathrm{~m}$. Now, for the sake of simplicity, let the layer from the surface down to $50 \mathrm{~cm}$ be the surface layer and the one below that be the bottom layer and assume that in each layer, $S$ and $B$ in the equation (1) are the same with each other. When the invasion coefficient is $\omega, \omega\left(S_{1}-S_{2}\right)$ is the amount of oxygen passing through the boundary of the two layers. In the surface layer, the equation (1) may be written as follows :

$$
d S^{\prime} / d t=\omega^{\prime}\left(s-S^{\prime}\right)+\omega^{\prime \prime}\left(S^{\prime \prime}-S^{\prime}\right)+B^{\prime}(t),
$$

and in the bottom layer :

$$
d S^{\prime \prime} / d t=\omega^{\prime \prime}\left(S^{\prime}-S^{\prime \prime}\right)+B^{\prime \prime}(t),
$$

where advection and convection can be neglected. In the above $s$ denotes the amount of saturated oxygen and $S^{\prime}$ and $S^{\prime \prime}$ are oxygen contents in the surface and the bottom layer respectively.

The following relations may be obtained from (2) and (3).

$$
S^{\prime} e^{2 \omega t}=S_{0}{ }^{\prime}+p^{\prime} \int_{0}^{t} I_{\varepsilon}{ }^{\prime} e^{2 \omega t} d t-c^{\prime} \int_{0}^{t} e^{k(\theta)-\bar{\theta})+2 \omega t} d t+\omega\left\{\int_{0}^{t} S e^{2 \omega t} d t+\int_{0}^{t} S^{\prime \prime} e^{2 \omega t} d t\right\},
$$




$$
S^{\prime \prime} e^{2 \omega t}=S_{0}^{\prime \prime}+p^{\prime \prime} \int_{0}^{t} I_{\varepsilon}^{\prime \prime} e^{(\omega t} d t-c^{\prime \prime} \int_{0}^{t} e^{k(\theta)-\bar{\theta})+\omega t} d t+\omega \int_{0}^{t} S^{\prime} e^{\omega t} d t
$$

where

$$
\omega^{\prime}=\omega^{\prime \prime}=\omega, \quad c=R_{1}+R_{2} ;
$$

and

$$
I_{\varepsilon}^{\prime}=I_{0} \cdot \varepsilon \cdot g e^{-k(\theta)-\bar{\theta})} \cdot\left(1-e^{-\beta a}\right), \quad I_{\varepsilon}^{\prime \prime}=I_{\varepsilon}^{\prime} e^{-k(\theta)-\bar{\theta})+k(\theta)-\bar{\theta})-\beta a} .
$$

$\vec{\theta}$ is the mean diurnal water temperature. $S_{0}$ is the oxygen content at sunrise. According to HARVEY [5], $\omega$ is in the order of magnitude of $10^{-3}$ to $10^{-5}$ in the CGS unit. Then, putting $\omega=10^{-4}$ and calculating on the assumption that $p^{\prime}=0.05$, $c^{\prime}=0.49, p^{\prime \prime}=0.05$ and $c^{\prime \prime}=0.44$, the caiculated curve may be obtained as shown in Fig. 2. Using the above values of $p$ and $c$, the amount of the oxygen consumption becomes $10 \sim 11 c c \mathrm{O}_{2} / l / d a y$ and the amount of the oxygen production becomes 0.5 $\sim 1.0$ cc $\mathrm{O}_{2} / l /$ day. The latter is the so-called net production.

The gross production amounts to 10.5 to 12.0 . These values vary with $\omega$. As $\omega$ becomes larger, the amplitude of the diurnal variation of oxygen becomes narrower, when $p, c$ and the effect of advection or convection are kept constant. Since the effect of advection or convection is not taken into account, $p$ and $c$ give predominant influences upon the amplitude when $\omega$ is constant. In other words, if the amplitude is kept constant, larger $\omega$ is accompanied with larger $p$ and $c$. Therefore, assuming the amplitude of diurnal variation of oxygen content to be constant, $p$ and $c$ take the least values when $\omega=0$. Thereby the corresponding production and consumption during a whole day will be determined.

The dotted lines in Figs. 3(a) and 6 show the computed results obtained by the same treatment as mentioned above. But, in these computation, $\omega$ was neglected.

\section{The time of the maximum and the minimum oxygen content}

Though the condition $\omega=0$ is not actually expected in nature, the assumption of it makes the equation simpler. Therefore, the approximation will be made on such an assumption. And comparison will be done between the calculated and the observed values. When $\omega=0$, the formula (4) becomes as follows:

$$
S-S_{0}=p \int_{0}^{t} I_{\varepsilon} d t-c \int_{0}^{t} e^{k(\theta-\bar{\theta})} d t .
$$

When the time distance equivalent to a whole day is denoted by $T$ and we put

then,

$$
\left(S_{T}-S_{0}\right) / c \int_{0}^{T} e^{k(\theta-\bar{\theta})} d t=\rho,
$$

$$
S-S_{0}=p\left[\int_{0}^{t} I_{\varepsilon} d t-\left\{\int_{0}^{t} e^{k(\theta-\bar{\theta})} d t \int_{0}^{r} I_{\varepsilon} d t / \int_{0}^{T} e^{k<\theta-\bar{\theta})}(1+\rho)\right\}\right],
$$

where $\tau$ shows the time distance between the sunrise and the sunset.

Since the maximum and the minimum appear at $t$ satisfying the equation $d S / d t=0$, the time at which the maximum or the minimum of oxygen content occurs 
may be obtained by the following equation :

$$
\varepsilon g(\cos Z)=\int_{0}^{\tau} \varepsilon g e^{k(\theta-\bar{\theta})} d t / \int_{0}^{T} e^{k<(\theta-\bar{\theta})} d t(1+\rho)
$$

The left side of (6) is a function of $\cos Z$ and

$$
\cos Z=\sin \varphi \sin \delta+\cos \phi \cos \delta \cos t^{\prime}
$$

where $\varphi$ : the latitude of the position of observation, $\delta$ : the sun's declination, $t^{\prime}:$ the sun's hour angle.

Since $g$ is a function of $\cos t^{\prime}, \cdots$ the maximum and the minimum of oxygen content occur at an equal time distance respectively before and after the noon. The time at which the maximum or the minimum occurs respectively in June and in December at Tokyo is as shown in the following table, if $\rho=0$.

The time of the maximum or the minimum oxygen content in the surface layers at Tokyo (calculated).

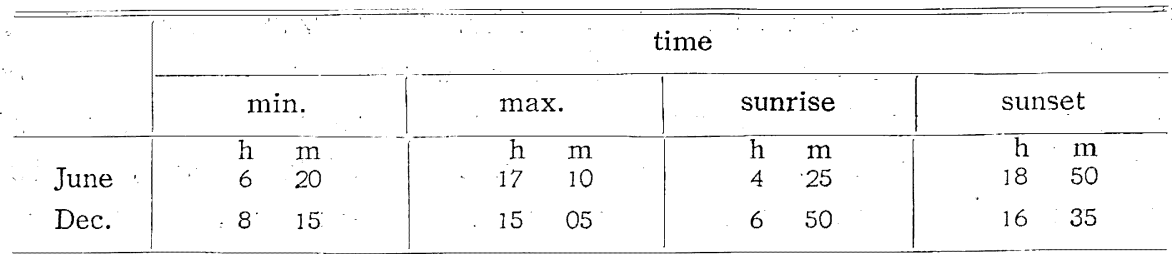

In this calculation, it is assumed, $\alpha[6]$ is 0.04 in June, 0.03 in December; $\beta$ [7]. is 0.04 in June, 0.03 in December. $\gamma$ corresponding to every altitude of the sun varies as given in the following table [8]:

\begin{tabular}{c|c|c|c|c|c|c}
\hline \hline Sun's altitude ( ${ }^{\circ}$ & 5 & 10 & 20 & 30 & 40 & $50-90$ \\
\hline$\gamma(\%)$ & 60 & 75 & 88 & 94 & 96 & 97 \\
\hline
\end{tabular}

$k(0.069)$ [9] represents the temperature coefficient of both photosynthesis and respiration. Because these activities increase twofold with temperature elevation by $10^{\circ} \mathrm{C}$. The value of $\varepsilon$ corresponding to any light intensity was obtained by Fig. 7. In Fig. 7 lux is used as the unit, but this must be exchanged into the energy unit such as $g$ cal. Though the relation between these two units is not simple and depends upon the wave-length of light, the following relation was adopted in this paper :

$$
\text { ig } \text { cal }=1200 \text { luxes [11]. }
$$

The calculated values given in the table do not shift by more than 30 minutes, even though $\rho$ may be estimated at 0.5 .

The maximum value was found at $11 \mathrm{~h}$ in Lake Suwa (Nagano Pref.) by J. Masuzawa [12] and at $22 \mathrm{~h}$ in the pond in front of our laboratory by the author and others*.

* This observation was made in cooperation with $\mathrm{K}$. SARUhashi, T. Ishibasil and K. IRIE under the direction of Dr. Y. Miyake. 
Such variations are due to the effect of advection or convection.

\section{The amplitude of the diurnal variation}

The amplitude of the diurnal variation is the difference between the maximum and the minimum amount of oxygen. But, for simplicity's sake the following amount is considered instead of the amplitude :

where

$$
\Delta S=S_{\tau}-S_{0}=p \int_{0}^{\tau} I_{\varepsilon} d t[1-\{q \tau /(1+\rho) T\}]
$$

$$
\int_{0}^{T} e^{k(\theta-\bar{\theta})} d t \doteqdot T, \quad q=\int_{0}^{\tau} e^{k(\theta-\bar{\theta})} d t / \tau .
$$

Depending on the cloudiness, $\int_{0}^{\tau} I_{\varepsilon} d t$ varies more remarkably than the value within the bracket. Hence, $\Delta S$ is mainly decided by the former.

Since $\Delta S$ is approximately proportional to the amplitude, the amplitude is larger on a clear day than on a cloudy day. But, the comparison of $\Delta S$ between two different seasons is not simple, because $p, I_{\varepsilon}$ and $\tau_{\tau}$ may take different values. Then, comparing each $\Delta S, \int_{0}^{\tau} I_{\varepsilon} d t$ and $\tau$ in the equation (7), the ratio of $p$ in two seasons may be obtained. In this manner, $p$ (June)/p(Dec.) was obtained from Kurashige's data shown in Fig. 6. If it is assumed that the values of $Q, a$ and $r$ of planktons are the same in June and in December, the ratio of $p$ will be the same as the ratio of $n$.

Then, $p$ (June) $/ p$ (Dec.) or $n$ (June) $/ n$ (Dec.) is 1.3... On the other hand, the observed value of this ratio was more than $1.6[13]$.

\section{The amount of the oxygen production and the consumption per day}

- The relation between the oxygen content and planktons-

When the following values obtained in the moat around the Imperial Palace are introduced into the equation (7),

$$
\Delta S=2 c c / l, \int_{0}^{\tau} I_{\varepsilon} d t=350 \mathrm{gcal}, \tau=16 \mathrm{hrs}, T=24 \mathrm{hrs} \text { and if } \rho=0.5 \text { and } q=1 \text {, then }
$$
$p$ is 0.01 .

Also, by the formula $c T=p \int_{0}^{r} I_{\varepsilon} d t /(1+\rho)$, the next relation can be obtained.

$$
c T=2.3 c c / l / d a y \text {. }
$$

This is the amount of the oxygen consumption per day. Then the net production of oxygen per day is given by

$$
\rho c T=1.2 c c / l / d a y \text {. }
$$

Accordingly, the gross production amounts to $3.5 c c / l / d a y$.

$$
\text { If } \rho=0, c T=6 c c / l / d a y \text {. }
$$

This is the amount of the gross production. 
By assuming that $r=10^{-3} \mathrm{~cm}, n=60 / \mathrm{cc}$ water and $Q \cdot a=0.3$. [14], $p$ can be computed as 0.01 by the equation $p=f Q a \dot{a} \pi r^{2} n$, where $n=60$ was given Dy E. KurAshige's data.

The gross production of oxygen per day is related to $\Delta S$. From the equation (7) the following relation is approximately obtained.

$$
(1+\rho) c T=T \Delta S /(T-\tau) \text {. }
$$

According to this equation, the gross production of oxygen per day is several times as much as $\Delta S$. Since $\Delta S$ is closely related to the amplitude of the diurnal variation, the gross production may be determined by the latter.

\section{Conclusions}

The author tried to explain the mechanism of the diurnal variation of dissolved oxygen from the physico- and biochemical standpoint. At first, a theoretical, generalized, formula was presented concerning the oxygen content in relation to the solar radiation, water temperature, number and size of living matter etc.

When the flow of water and the effect of diffusion are negligible, the formula becomes simpler. By this simplified formula, it was concluded that the minimum and the maximum of dissolved oxygen appeared at an equal time distance respectively before and after the noon. Then, in Tokyo, the minimum should occur at $6 \sim 8 \mathrm{~h}$ and the maximum at 15 17h. The amplitude of diurnal variation is larger on a clear day than on a cloudy day and also it is larger in summer than in winter. These facts could be explained theoretically. Also, the amount of the oxygen production and consumption during a day was computed, assuming the number and the size of phytoplanktons. These values agree well with those observed by $\mathrm{E}$. Kurashige.

\section{References}

[1] Kurashige, E., 1932: The Diurnal Change of Dissolved Oxygen in Relacion to the Solar Radiation in the Ohori (Moat), Part IT. Journal of the Meteorological Society of Japan (in Japanese), II, 10, p. 489.

[2] Mryake, Y., 1948: The Diurnal Variation of Dissolved Oxygen in Sea Water Consisting of Different Water Masses. The Geophysical Magazine, 16, p. 66.

[3] Mryake, Y., Suglura, Y. and Kameda, K., 1953: A Study on the Property of the Coastal Waters around Hachijo Island. Records of Oceanographic Works in Japan, 1, p. 97.

[4] Kurashige, E., 1933: Diurnal Change of Dissolved $\mathrm{O}_{2}, \mathrm{CO}_{2}$ and $\mathrm{pH}$ in the Water of Ohori (Castle-moat) in Relation to the Solar Radiation, Part III (Their Seasonal Fluctuation). Journal of the Meteorological Society of Japan (in Japanese), II, 11, p. 253. 
[5] Harvey, H.W., 1928: Biological Chemistry and Physics of Sea Water. Cambridge, the University Press, p. 61.

[6] Kitaoka, T. and Matuoka, Y., 1944: On the "Trübungsfactor" of the Atmosphere in Japan, Observed with Silverdisc Pyrheliometer. Journal of the Meteorological Society of Japan (in Japanese), II, 22, p. 64.

[7] Takenouchi, Y., 1939: On the Under Water Illuminations. Bulletin of the Japanese Society of Scientinac Fisheries (in Japanese), 8, p. 204.

[8] Sverdiup, H.U., Johnson, M.W. and Fleming, R.H., 1952: The Oceans. New York, Prentice-Hall, p. 104.

[9] Riley, G.A. and ARx, R. V., 1942: Theoretical Analysis of Seasonal Changes in the Phytoplanktons of Pusan Harbour, Korea. Journal of the Marine Research, 7, p. 60.

[10] Nielsen, E.S., 1952: The Use of Radio-active Carbon $\left(\mathrm{C}^{14}\right)$ for Measuring Organic Production in the Sea. Journal du Conseil, 18, p. 131.

[11] Sverdrup, H.U., Johnson, M.W. and Fleming, R.H., 1952: The Oceans. New York, Prentice-Hall, p. 775.

[12] Masuzawa, J.: Kaki ni Okeru Kosho no Seiso Henka to Koshoseisan-ryoku (On Change of the Stratified Structure and Plant Production of a Lake in Summer), Oyo kisho (in Japanese); 1, No. 3, p. 1.

[13] Sakamura, T., 1947: Shokubutsu Seirigaku (Plant Physiology) (in Japanese). Tokyo, p. 240 .

[14] Kurashige, E., 1932: Quantitative Investigation on the Seasonal Distribution of Phytoplankton in the Ohori (Moat). Journal of the Meteorological Society of Japan (in Japanese), II, 10, p. 477. 